\title{
The early afterglow and magnetized ejecta present in GRB 110731A
}

\author{
Nissim Fraija ${ }^{* \dagger}$ \\ Instituto de Astronomía, UNAM \\ E-mail: nifraija@astro.unam.mx
}

\section{William H. Lee}

Instituto de Astronomía, UNAM

E-mail: wlee@astro.unam.mx

One of the most energetic gamma-ray bursts GRB 110731A, was observed from optical to GeV energy range by Fermi and Swift Observatories, and by the MOA and GROND optical telescopes. The multiwavelength observations over different epochs (from trigger time to more than $800 \mathrm{~s}$ ) showed that the spectral energy distribution was better fitted by a wind afterglow model. We present a leptonic model based on an early afterglow that evolves in a stellar wind to describe the multiwavelength light curves observations. In particular, the origin of the LAT emission is explained through the superposition of synchrotron radiation from the forward shock and synchrotron self-Compton emission from the reverse shock. The bulk Lorentz factor required in this model is $\Gamma \simeq 520$ and the result suggests that the ejecta must be magnetized.

Swift: 10 Years of Discovery,

2-5 December 2014

La Sapienza University, Rome, Italy

\footnotetext{
* Speaker.

${ }^{\dagger}$ Luc Binette postdoctoral scholarship.
} 


\section{Introduction}

In recent years, the detection of $\gamma$-ray and optical polarization in gamma-ray bursts (GRBs) has supported the idea that jets could be magnetized [1]. The jet evolution with magnetic content has been explored in several contexts. In these models, an electromagnetic component is introduced through the magnetization parameter $(\sigma)$ and defined by the ratio of Poynting flux (electromagnetic component) and matter energy (internal+kinetic component) $[2,3]$.

The afterglow phase is one of the most interesting and least understood episodes of the burst. During this episode, the relativistic ejecta interacts with the surrounding matter generating reverse and forward shocks. In a wind and homogeneous environment, the reverse shock has been discussed to describe the early $\gamma$-ray, optical and/or radio flares present in some bursts $[4,5,6,7]$ and the forward shock to explain the continuous softening of the afterglow spectrum [8].

Hadronic (inelastic proton-neutron collisions and proton-photon interactions) and leptonic (inverse Compton (IC), synchrotron self-Compton (SSC) and synchrotron emission) models have been widely explored to explained the photons observed with energies $\geq 100 \mathrm{MeV}[9,10,11]$.

The bright and long GRB 110731A was observed in $\gamma$-ray, X-ray and optical wavelengths. The analysis of the prompt phase revealed an extremely bright peak and a temporally extended component in the Large Area Telescope (LAT) light curve starting at $\sim 5.5 \mathrm{~s}$ [12]. In addition, temporal and spectral analysis in different wavelengths and epochs (just after the trigger time and extending for more than $800 \mathrm{~s}$ ) favored a wind afterglow model. Recently, Lemoine et al. (2013) [13] proposed that if the magnetic equipartition parameter would vary as a function of time, $\varepsilon_{B} \propto t^{-\alpha_{t}}$ with $0.5 \leq \alpha_{t} \leq 0.4$, then the $\mathrm{GeV}$ photons would be likely created in a region of strong $\varepsilon_{B}$. They argued that the magnetization that permeates the blast wave of GRB 110731A could be described as partial decay of the micro-turbulence [14] as observed in particle-in-cell (PIC) simulations [15]. In this paper, we develop a leptonic model based on external shocks (forward and reverse) that evolves adiabatically in a stellar wind. We show that with the suitable equipartition parameters we are able to reproduce the LAT, X-ray and optical light curves (LCs) observed in GRB 110731A.

\section{GRB 110731A}

GRB 110731A was localized with coordinates R. A. $=18^{h} 41^{m} 00^{s}$ and dec. $=-28^{\circ} 31^{\prime} 00^{\prime \prime}$ (J2000), with a $68 \%$ confidence error radius of $0.2^{\circ}$.

This burst was detected by the Gamma-Ray Burst Monitor and LAT [12] on board Fermi; BAT, XRT and UVOT [16] on board Swift, and the Microlensing Observations in Astrophysics (MAO) telescope [17] and Gamma- ray Burst Optical/Near-Infrared Detector (GROND). LAT began to observe this burst from $\sim 4 \mathrm{~s}$ after trigger time to more than $800 \mathrm{~s}$, detecting an extremely bright peak at $5.5 \mathrm{~s}$. Swift/BAT perceived immediately this burst after the detection by both instruments of Fermi, whereas XRT and UVOT began observations 56s after the BAT trigger. UVOT swiftly determined the afterglow position as R.A. $=18^{h} 42^{m} 00^{s} .99$ and dec. $=-28^{\circ} 32^{\prime} 13^{\prime \prime} .8$ (J2000), with a $90 \%$ confidence. The lack of observation in the UV filters is consistent with the measured redshift $\mathrm{z}=2.83$ [17]. MAO observations started 3.3 minutes after the Swift trigger and GROND mounted on the $2.2 \mathrm{~m} \mathrm{MPG/ESO} \mathrm{telescope} \mathrm{at} \mathrm{La} \mathrm{Silla} \mathrm{Observatory,} \mathrm{Chile,} \mathrm{observed} \mathrm{this} \mathrm{burst} 2.74$ days after 
the trigger [18].

\section{Wind-afterglow Model}

Afterglow hydrodynamics involves a relativistic blast wave expanding into the medium with density

$$
\rho=A r^{-2} \text { with } A=\frac{\dot{M}_{w}}{4 \pi V_{w}},
$$

where $\dot{M}_{w}$ is the mass loss rate and $V_{w}$ is the wind velocity. We hereafter use primes (unprimes) to define the quantities in a comoving (observer) frame and $\mathrm{c}=\hbar=1$ in natural units. The radius shock $(r)$ spreading into this density can be written in the form

$$
r=\frac{3 \xi}{2 \pi^{1 / 2}}(1+z)^{-1 / 2} E^{1 / 2} t^{1 / 2} A^{-1 / 2}
$$

where the total energy of the shock is $E=8 \pi / 9 A \Gamma^{2} r$ with $\Gamma$ the bulk Lorentz factor, $\xi$ a constant parameter [21], $z$ the redshift and $t=(1+z) \frac{r}{4 \xi^{2} \Gamma^{2}}$ is the time in the observer's frame.

By considering the typical values of the stellar wind $\left(A=A_{\star} \times\left(5.0 \times 10^{11}\right) \mathrm{g} / \mathrm{cm}\right.$ with $A_{\star}=$ 0.1 ; [20], the parameter $\xi=0.56$; [21]) and those inferred by observations: redshift $z=2.83$ [17], total energy $E \simeq 10^{54} \mathrm{erg}$ and duration of GRB $T_{90}=7.3 \mathrm{~s}$, we calculate the SSC and synchrotron spectral breaks from forward and reverse shocks. The subscripts $f$ and $r$ refer throughout this paper to the forward and reverse shocks, respectively.

\subsection{Forward Shocks}

By considering the brightest peak of the flux density present at the end of the prompt emission, in the interval $[5.47 \mathrm{~s}, 5.67 \mathrm{~s}]$, and also the LAT flux decaying smoothly during the whole temporally extended emission, we constrain the Lorentz factor $\Gamma \simeq 520$ so that the deceleration time occurs at

$$
t_{d e c} \simeq 5.55 \mathrm{~s}\left(\frac{1+z}{4}\right) E_{54} A_{\star,-1}^{-1} \Gamma_{2.72}^{-4} .
$$

We consider that electrons are accelerated in the shock to a power-law distribution $N\left(\gamma_{e}\right) d \gamma_{e} \propto$ $\gamma_{e}^{-p} d \gamma_{e}$ with a minimum Lorentz factor $\gamma_{e} \leq \gamma_{m}=\varepsilon_{e, f}(p-2) /(p-1) m_{p} / m_{e} \Gamma$, where $\varepsilon_{B}=B^{2} /\left(32 \pi \Gamma^{2} \rho\right)$ and $\varepsilon_{e}=U_{e} /\left(4 \Gamma^{2} \rho\right)$ are the magnetic and electron equipartition parameters, respectively, and the magnetic field is $B_{f}^{\prime} \simeq \frac{8 \sqrt{2} \pi}{3 \xi}(1+z)^{1 / 2} \varepsilon_{B, f}^{1 / 2} \Gamma E^{-1 / 2} t^{-1 / 2} A$. Comparing the cooling $t_{e, s y n} \simeq 3 m_{e} /\left(16 \sigma_{T}\right)(1+$ $\left.x_{f}\right)^{-1}(1+z) \varepsilon_{B, f}^{-1} \rho^{-1} \Gamma^{-3} \gamma_{e}^{-1}$ and acceleration $t_{a c c} \simeq \frac{2 \pi m_{e}}{q_{e}}(1+z) \Gamma^{-1} B_{f}^{\prime-1} \gamma_{e}$ time scales with the deceleration time (eq. 3.3), we obtain the cooling $\gamma_{e, c, f}=\frac{3 m_{e} \xi^{4}}{\sigma_{T}}\left(1+x_{f}\right)^{-1}(1+z)^{-1} \varepsilon_{B, f}^{-1} \Gamma A^{-1} t$ and maximum $\gamma_{e, \max , f} \simeq \sqrt{\frac{9 \sqrt{2} q_{e}}{16 \pi \sigma_{T}}} \xi^{1 / 2}(1+z)^{-1 / 4} \varepsilon_{B, f}^{-1 / 4} \Gamma^{-1 / 2} E^{1 / 4} A^{-1 / 2} t^{1 / 4}$ electron Lorentz factors. With the previous relations, we can write the synchrotron spectral breaks as

$$
E_{\gamma, \mathrm{a}, \mathrm{f}}^{s y n} \simeq 5.56 \times 10^{-4} \mathrm{eV}\left(\frac{1+z}{4}\right)^{-2 / 5} \varepsilon_{e, f,-0.4}^{-1} \varepsilon_{B, f,-4.15}^{1 / 5} A_{\star,-1}^{6 / 5} E_{54}^{-2 / 5} t_{1}^{-3 / 5}
$$




$$
\begin{aligned}
E_{\gamma, \mathrm{m}, \mathrm{f}}^{s y n} & \simeq 77.45 \mathrm{keV}\left(\frac{1+z}{4}\right)^{1 / 2} \varepsilon_{e, f,-0.4}^{2} \varepsilon_{B, f,-4.15}^{1 / 2} E_{54}^{1 / 2} t_{1}^{-3 / 2} \\
E_{\gamma, \mathrm{c}, \mathrm{f}}^{s y n} & \simeq 0.30 \mathrm{eV}\left(\frac{1+z}{4}\right)^{-3 / 2}\left(\frac{1+x_{f}}{11}\right)^{-2} \varepsilon_{B, f,-4.15}^{-3 / 2} A_{\star,-1}^{-2} E_{54}^{1 / 2} t_{1}^{1 / 2} \\
E_{\gamma, \text { max }, \mathrm{f}}^{s y n} & \simeq 36.94 \mathrm{GeV}\left(\frac{1+z}{4}\right)^{-3 / 4} E_{54}^{1 / 4} A_{\star,-1}^{-1 / 4} t_{1}^{-1 / 4} .
\end{aligned}
$$

Here $\sigma_{T}$ is the Thomson cross section, $m_{e}$ is electron mass, $q_{e}$ is the elementary charge, the term $\left(1+x_{f}\right)$ is introduced as a correction factor and $D$ is the luminosity distance. The transition time from fast- to slow-cooling regime is $t_{0}^{\text {syn }}=123.46 s\left(\frac{1+z}{4}\right) \varepsilon_{e, f,-0.4} \varepsilon_{B, f,-4.15} A_{\star,-1}$. It is worth noting that X-ray and optical fluxes can be described through the spectral evolution of the characteristic $E_{\gamma, \mathrm{m}, \mathrm{f}}^{s y n} \simeq 77.45 \mathrm{keV}$ and cooling $E_{\gamma, \mathrm{c}, \mathrm{f}}^{s y n} \simeq 0.30 \mathrm{eV}$ break energies and after $\sim 100 \mathrm{~s}$ the synchrotron spectrum changes from fast to slow regime. From the maximum photon energy $E_{\gamma, \text { max }, \mathrm{f}}^{s y n} \simeq 36.94 \mathrm{GeV}$, one can see that the temporally extended LAT component can be explained by synchrotron emission of high-energy electrons radiating at $\simeq 100 \mathrm{MeV}$. Fermi-accelerated electrons may scatter synchrotron photons up to higher energies as $E_{\gamma, i}^{s s c} \simeq 2 \gamma_{e, i}^{2} E_{\gamma, i}^{s y n}$. From the synchrotron spectral breaks (eqs. 3.4), the spectral breaks in the Compton regime are

$$
\begin{aligned}
E_{\gamma, \mathrm{m}, \mathrm{f}}^{s s c} & \simeq 11.66 \mathrm{TeV}\left(\frac{1+z}{4}\right) \varepsilon_{e, f,-0.4}^{4} \varepsilon_{B, f,-4.15}^{1 / 2} A_{\star,-1}^{-1 / 2} E_{54} t_{2}^{-2} \\
E_{\gamma, \mathrm{c}, \mathrm{f}}^{s s c} & \simeq 162.8 \mathrm{keV}\left(\frac{1+z}{4}\right)^{-3}\left(\frac{1+x_{f}}{11}\right)^{-4} \varepsilon_{B, f,-4.15}^{-7 / 2} A_{\star,-1}^{-9 / 2} E_{54} t_{2}^{2} \\
F_{\gamma, \text { max } \mathrm{f}}^{s s c} & \simeq 0.41 \mathrm{Jy}\left(\frac{1+z}{4}\right) \varepsilon_{B, f,-4.15}^{1 / 2} A_{\star,-1}^{1 / 2} D_{28}^{-2} E_{54},
\end{aligned}
$$

where the break energy at the Klein-Nishina regime is $E_{\gamma, f}^{K N}=42.33 \mathrm{GeV}$. The value of equipartition parameters $\varepsilon_{B, f}=10^{-4.15}$ and $\varepsilon_{e, f}=0.4$ were obtained after fitting the multiwavelength LC observations (see [19]). We can see that the maximum photon energy achieved by synchrotron radiation is $36.94 \mathrm{GeV}$ and the characteristic SSC spectral break is $11.66 \mathrm{TeV}$, therefore the photon with energy of $3.4 \mathrm{GeV}$ detected at hundreds of seconds [12] could be explained by the synchrotron and/or SSC emission.

\subsection{Reverse shocks}

The critical Lorentz factor can be written as

$$
\Gamma_{c} \simeq 472.5\left(\frac{1+z}{4}\right)^{1 / 4} A_{\star,-1}^{-1 / 4} E_{54}^{1 / 4}\left(\frac{T_{90}}{7.3 s}\right)^{-1 / 4} .
$$

Since the brightest peak was present in the interval $[5.47 \mathrm{~s}, 5.67 \mathrm{~s}$ ], we consider that bulk Lorentz factors at forward and reverse shocks are equal $\Gamma \simeq 520>\Gamma_{c}$, then the reverse shock evolves in the thick-shell case. Synchrotron spectral breaks between forward and reverse shocks are related by [4]

$$
E_{\gamma, \mathrm{m}, \mathrm{r}}^{s y n} \sim \mathscr{R}_{e}^{2} \mathscr{R}_{B}^{-1 / 2} \mathscr{R}_{M}^{-2} E_{\gamma, \mathrm{m}, \mathrm{f}}^{s y n}, \quad E_{\gamma, \mathrm{c}, \mathrm{r}}^{s y y} \sim \mathscr{R}_{B}^{3 / 2} \mathscr{R}_{x}^{-2} E_{\gamma, \mathrm{c}, \mathrm{f}}^{s y n}, \quad F_{\gamma, \max , \mathrm{r}}^{s y n} \sim \mathscr{R}_{B}^{-1 / 2} \mathscr{R}_{M} F_{\gamma, \max , f}^{s y n}
$$


where $\mathscr{R}_{B}=\frac{\varepsilon_{B, f}}{\varepsilon_{B, r}}, \mathscr{R}_{e}=\frac{\varepsilon_{e, r}}{\varepsilon_{e, f}}, \mathscr{R}_{x}=\frac{1+x_{f}}{1+x_{r}+x_{r}^{2}}$ and $\mathscr{R}_{M}=\frac{\Gamma_{c}^{2}}{\Gamma}$. From eqs. (3.4, 3.7 and 3.6), we get that the synchrotron break energies can be written as

$$
\begin{aligned}
E_{\gamma, \mathrm{a}, \mathrm{r}}^{s y n} & \simeq 4.28 \times 10^{-8} \mathrm{eV}\left(\frac{1+z}{4}\right)^{-7 / 5} \varepsilon_{e, r,-0.4}^{-1} \varepsilon_{B, r,-0.55}^{1 / 5} \Gamma_{2.72}^{2} A_{\star,-1}^{11 / 5} E_{54}^{-7 / 5}\left(\frac{T_{90}}{7.3 s}\right)^{2 / 5} \\
E_{\gamma, \mathrm{m}, \mathrm{r}}^{s y n} & \simeq 128.94 \mathrm{eV}\left(\frac{1+z}{4}\right)^{-1 / 2} \varepsilon_{e, r,-0.4}^{2} \varepsilon_{B, r,-0.55}^{1 / 2} \Gamma_{2.72}^{2} A_{\star,-1} E_{54}^{-1 / 2}\left(\frac{T_{90}}{7.3 s}\right)^{-1 / 2} \\
E_{\gamma, \mathrm{c}, \mathrm{r}}^{s y n} & \simeq 0.93 \times 10^{-5} \mathrm{eV}\left(\frac{1+z}{4}\right)^{-3 / 2}\left(\frac{1+x_{r}+x_{r}^{2}}{3}\right)^{-2} \varepsilon_{B, r,-0.55}^{-3 / 2} A_{\star,-1}^{-2} E_{54}^{1 / 2}\left(\frac{T_{90}}{7.3 s}\right)^{1 / 2} \\
F_{\gamma, \text { max }, \mathrm{r}} & \simeq 4.31 \times 10^{4} \mathrm{Jy}\left(\frac{1+z}{4}\right)^{2} \varepsilon_{B, r,-0.55}^{1 / 2} \Gamma_{2.72}^{-1} A_{\star,-1}^{1 / 2} D_{28}^{-2} E_{54}\left(\frac{T_{90}}{7.3 s}\right)^{-1} .
\end{aligned}
$$

As one can see the synchrotron self-absorption is in the weak absorption regime $\left(E_{\gamma, a, r}^{s y n}<E_{\gamma, c, r}^{s y n}\right)$, hence there is not a thermal component besides the power-law spectrum. The synchrotron emission from reverse shock predicts a peak of the flux density at $\sim 100 \mathrm{eV}$. On the other hand, accelerated electrons can upscatter photons from low to high energies as $E_{\gamma, \mathrm{a}, \mathrm{r}}^{\mathrm{ssc}} \sim 2 \gamma_{e, m, r}^{2} E_{\gamma, \mathrm{a}, \mathrm{r}}^{s y n}, E_{\gamma, \mathrm{m}, \mathrm{r}}^{\mathrm{ssc}} \sim 2 \gamma_{e, m, r}^{2} E_{\gamma, \mathrm{m}, \mathrm{r}}^{s y n}$, $E_{\gamma, \mathrm{c}, \mathrm{r}}^{s s c} \sim 2 \gamma_{\mathrm{e}, \mathrm{c}, \mathrm{r}}^{2} E_{\gamma, c, r}^{s y n}$ and $F_{\gamma, \text { max }, \mathrm{r}}^{s s c} \sim k \tau F_{\gamma, \text { max }, \mathrm{r}}^{s y}$ where $k=4(p-1) /(p-2), \tau=\frac{\sigma_{T} N\left(\gamma_{e}\right)}{4 \pi r_{d}}$ is the optical depth of the shell and $N_{e}$ is the number of radiating electrons. From eq. (3.8), SSC spectral breaks can be written as

$$
\begin{aligned}
E_{\gamma, \mathrm{m}, \mathrm{r}}^{s s c} & \simeq 103.55 \mathrm{MeV}\left(\frac{1+z}{4}\right)^{-1} \varepsilon_{e, r,-0.4}^{4} \varepsilon_{B, r,-0.55}^{1 / 2} \Gamma_{2.72}^{4} A_{\star,-1}^{3 / 2} E_{54}^{-1}, \\
E_{\gamma, \mathrm{c}, \mathrm{r}}^{s s c} & \simeq 5.86 \times 10^{-3} \mathrm{eV}\left(\frac{1+z}{4}\right)^{-3 / 2}\left(\frac{1+x_{r}+x_{r}^{2}}{3}\right)^{-4} \varepsilon_{B, r,-0.55}^{-7 / 2} \Gamma_{2.72}^{-6} A_{\star,-1}^{-6} E_{54}^{5 / 2}\left(\frac{T_{90}}{7.3 s}\right)^{1 / 2}, \\
F_{\gamma, \text { max }, \mathrm{r}}^{s s c} & \simeq 1.42 \times 10^{2} \mathrm{Jy}\left(\frac{1+z}{4}\right)^{3} \varepsilon_{B, r,-0.55}^{1 / 2} \Gamma_{2.72}^{-2} A_{\star,-1}^{3 / 2} D_{28}^{-2} E_{54}\left(\frac{T_{90}}{7.3 s}\right)^{-2}
\end{aligned}
$$

From SSC LCs in the fast-cooling regime of reverse shock [7], eq. (3.9) and the crossing time $\sim T_{90} / 6$, one can describe the brightest LAT peak as SSC emission from reverse shock. In fact, the value of equipartition parameters $\varepsilon_{B, r}=0.28$ and $\varepsilon_{e, r}=0.4$ were obtained after fitting this peak at $5.5 \mathrm{~s}$ (see [19]).

\section{Conclusions}

We have presented a leptonic model based on the evolution of an early afterglow in the stellar wind. The current model having six free parameters (bulk Lorentz factor, density of the stellar wind, electron and magnetic equipartition parameters) accounts for the main temporal and spectral characteristics of GRB 110731A. The LAT emission extended up to $853 \mathrm{~s}$ is interpreted as synchrotron emission from forward shock and the brightest LAT peak is described evoking the SSC emission from reverse shock. Additionally, X-ray and optical fluxes are naturally explained by synchrotron emission from forward shock. We consider that the ejecta propagating in the stellar wind is early decelerated at $\sim 5.5 \mathrm{~s}$ and the reverse shock evolves in the thick-shell regime. Taking into account the values of redshift $z=2.83$ [17], energy $E \simeq 10^{54} \mathrm{erg}$, duration of GRB $T_{90}=7.3 \mathrm{~s}[12,16]$ and the stellar wind $A=5.0 \times 10^{10} \mathrm{~g} / \mathrm{cm}[20]$, we get that the value of the Lorentz factor is $\Gamma \simeq 520$.

Comparing the magnetic equipartition parameters that best describe the emission at forward and 
reverse shocks, we can see that magnetic fields in both shocks are related by $B_{f} \simeq 2 \times 10^{-2} B_{r}$. The previous result as found in other bursts $[4,5,6]$ illustrates that the magnetic field in the reverse-shock region is stronger ( $\sim 50$ times) than in the forward-shock region which indicates that the ejecta is magnetized. Although from this burst photons at sub-TeV energies were not observed, in other bursts they might be present. In this case, these sub-TeV could be modeled as SSC emission from forward shock and also be candidates to be detected by $\mathrm{TeV} \gamma$-ray observatories as the High Altitude Water Cherenkov observatory (HAWC) [22].

\section{References}

[1] W. Coburn, and S. E. Boggs, Polarization of the prompt $\gamma$-ray emission from the $\gamma$-ray burst of 6 December 2002, Nature 423 (2003) 415

[2] G. Drenkhahn, Acceleration of GRB outflows by Poynting flux dissipation, A\&A 387 (2002) 714

[3] N. Vlahakis and A. Königl, Relativistic Magnetohydrodynamics with Application to Gamma-Ray Burst Outflows. II. Semianalytic Super-Alfvénic Solutions, ApJ 596 (2003) 1104

[4] S. Kobayashi and B. Zhang, Early Optical Afterglows from Wind-Type Gamma-Ray Bursts, ApJ 597 (2003) 455

[5] N. Fraija, M. M. González and W. H. Lee, Synchrotron Self-Compton Emission as the Origin of the Gamma-Ray Afterglow Observed in GRB 980923, ApJ 751 (2012) 233

[6] J. R. Sacahui, N. Fraija, M. M. González and W. H. Lee,, The Long and the Short of the High-energy Emission in GRB090926A: An External Shock, ApJ 755 (2012) 127

[7] Z.-Y. Li and R. A. Chevalier, Wind-Interaction Models for the Early Afterglows of Gamma-Ray Bursts: The Case of GRB 021004, ApJL 589 (2003) L69

[8] A. Panaitescu, Swift gamma-ray burst afterglows and the forward-shock model, MNRAS 379 (2007) 331

[9] C. D. Dermer and A. Atoyan, Neutral beam model for the anomalous $\gamma$-ray emission component in GRB 941017, A\&A 418 (2004) L5

[10] R. Sari and A. A. Esin, On the Synchrotron Self-Compton Emission from Relativistic Shocks and Its Implications for Gamma-Ray Burst Afterglow, ApJ 548 (2001) 787

[11] B. Zhang and P. Mészáros, High-Energy Spectral Components in Gamma-Ray Burst Afterglows ApJ, $\mathbf{5 5 9}(2001) 110$

[12] M. Ackermann and et al., Multiwavelength Observations of GRB 110731A: GeV Emission from Onset to Afterglow, ApJ 763 (2013) 71

[13] M. Lemoine, Z. Li and X. Y. Wang, On the magnetization of gamma-ray burst blast waves, MNRAS 435 (2013) 3009

[14] E. Rossi and M. J. Rees, Gamma-ray burst afterglow emission with a decaying magnetic field, MNRAS 339 (2003) 881

[15] L. Sironi and A. Spitkovsky, Particle Acceleration in Relativistic Magnetized Collisionless Electron-Ion Shocks, ApJ 726 (2011) 75

[16] S. R. Oates et al., Swift Observations of GRB 110731A, GCN Report 343 (2011) 1

[17] N. R. Tanvir et al., GRB 110731A Gemini-N redshift, GRB Coordinates Network 12225 (2011) 1 
[18] J. Greiner et al., PGROND a 7-Channel Imager, PASP 120 (2008) 405

[19] N. Fraija, GRB 110731A: Early Afterglow in Stellar Wind Powered By a Magnetized Outflow, ApJ 804 (2015) 105

[20] R. A. Chevalier and X. Y. Li, Wind Interaction Models for Gamma-Ray Burst Afterglows: The Case for Two Types of Progenitors, ApJ 536 (2000) 195

[21] A. Panaitescu and P. Mészáros, Rings in Fireball Afterglows, ApJL 493 (1998) L31

[22] A. U. Abeysekara et al., Search for Gamma-Rays from the Unusually Bright GRB 130427A with the HAWC Gamma-Ray Observatory, ApJ 800 (2015) 78 